\title{
Synthesis of oleophilic electron-rich phenylhydrazines
}

Aleksandra Jankowiak ${ }^{1}$ and Piotr Kaszyński ${ }^{* 1,2}$

\section{Full Research Paper}

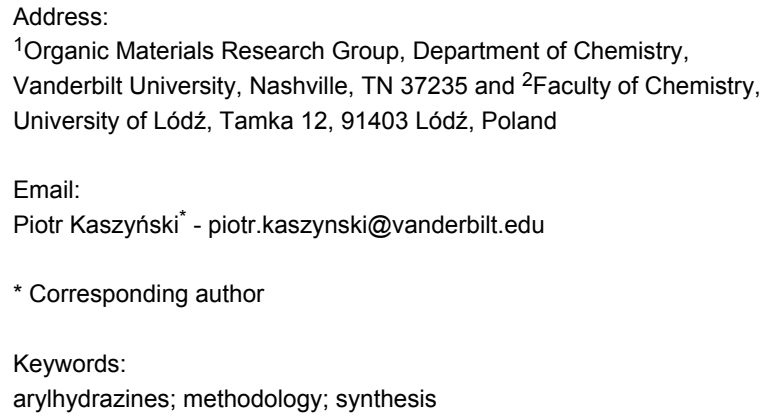

\begin{abstract} late (DTBAD).

\section{Introduction}

Mono-arylhydrazines I are important intermediates in the synthesis of a number of heterocycles, including indoles [1] and some azoles (for example [2,3]), many of which exhibit biological activity and are used in drug development [4-6]. Arylhydrazines are also key intermediates in the preparation of stable radicals such as verdazyl [7-9] and benzo[1,2,4]triazinyls [10$12]$.
\end{abstract}

Phenylhydrazines 1 substituted with two or three long-chain alkyl, alkoxy or alkylsulfanyl groups were successfully prepared by acid-induced removal of the Boc group in hydrazides 2 . The reaction is carried out with 5 equivalents of $\mathrm{TfOH}_{\mathrm{f}} \mathrm{CF}_{3} \mathrm{CH} \mathrm{H}_{2} \mathrm{OH} /$ $\mathrm{CH}_{2} \mathrm{Cl}_{2}$ at $-40{ }^{\circ} \mathrm{C}$ for $1.5 \mathrm{~min}$. Under these conditions, the deprotected hydrazine $\mathbf{1}$ is fully protonated, which increases its stability in the reaction medium. The hydrazines were isolated in $60-86 \%$ yields and purities $>90 \%$. The hydrazides 2 were obtained in $43-71 \%$ yields from aryl bromides 5 , which were lithiated with $t$-BuLi and subsequently reacted with di-tert-butyl azodicarboxy-

The parent phenylhydrazine and many of its electron-deficient derivatives, such as $p$-nitrophenylhydrazine, are stable under ambient conditions and are conveniently obtained by using classical methods, such as the reduction of diazonium salts [13-15].
In contrast, electron-rich arylhydrazines are far less numerous and their preparation is complicated by oxidative instability.

To access functionalized and sensitive arylhydrazines several methods involving the deprotection of hydrazides II have been developed (Figure 1). Hydrazides II are efficiently obtained by the addition of organometallic reagents III, prepared from aryl halide IV, to azodicarboxylate diesters (AD) $[16,17]$. Alternatively, II can be obtained in the $\operatorname{Pd}(0)$ - or $\mathrm{Cu}^{2+}$-catalyzed reaction of arylboronic acid V to AD [18-20]. The latter method is especially suited for arylhydrazides substituted with sensitive functional groups. Protected electron-rich arylhydrazines, 


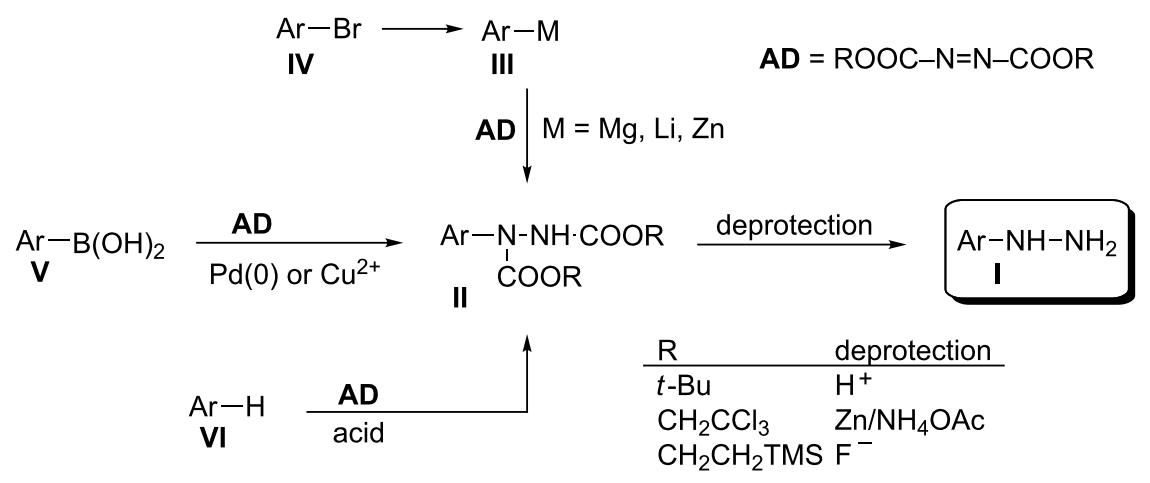

Figure 1: Selected methods for the preparation of arylhydrazines I through hydrazides II.

hydrazides II, containing the 2,2,2-trichloroethyl group $(\mathrm{R}=$ $\mathrm{CH}_{2} \mathrm{CCl}_{3}$ ) are conveniently prepared by direct electrophilic amination of arenes VI with bis(2,2,2-trichloroethyl) azodicarboxylate (BTCEAD) under Lewis [21,22] or Brønsted [23] acid conditions.

By judicious choice of the substituent $\mathrm{R}$, the removal of the protecting group in II and formation of arylhydrazines I can be accomplished under acidic $(\mathrm{R}=t$ - $\mathrm{Bu})[16]$, reductive $(\mathrm{R}=$ $\mathrm{CH}_{2} \mathrm{CCl}_{3}$ ) [24], or nearly neutral ( $\mathrm{R}=\mathrm{CH}_{2} \mathrm{CH}_{2} \mathrm{TMS}$ ) conditions $[22,25]$. Among the three methods, the most straightforward is the removal of the Boc group under acidic conditions. Unfortunately, the literature method for deprotection $(\mathrm{HCl}$ in isopropanol, $70^{\circ} \mathrm{C}$ ) has limited scope, and electron-rich 3,4dimethoxyphenylhydrazine could not be obtained under these conditions, although 4-pentyloxyphenylhydrazine hydrochloride was isolated in $60 \%$ yield [16]. The controlled reduction of 2,2,2-trichloroethyl esters ( $\mathbf{I I}, \mathrm{R}=\mathrm{CH}_{2} \mathrm{CCl}_{3}$ ) with $\mathrm{Zn}$ in aqueous $\mathrm{MeOH}$ containing $\mathrm{NH}_{4} \mathrm{OAc}$ gave access to a number of small, electron-rich phenylhydrazines, including 3,4dimethoxyphenylhydrazine isolated in $76 \%$ yield as hydrochloride [24].

In the context of our research program in liquid-crystalline verdazyl derivatives [26], we needed phenylhydrazines 1

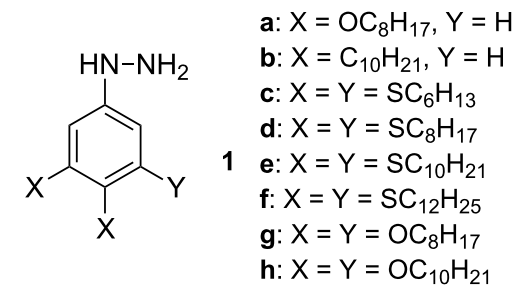

Figure 2: The structures for hydrazines $1 \mathrm{a}-\mathbf{1 h}$.
(Figure 2) substituted with multiple long-chain alkyl, alkoxy and alkylsulfanyl groups. Here we demonstrate an efficient method for the preparation of several hydrophobic di- and trisubstituted phenylhydrazines in purities sufficient for further chemical transformations. Finally, we demonstrate the application of one of the phenylhydrazines for the preparation of a discotic liquid crystal.

\section{Results and Discussion}

Our initial attempts at the preparation of 3,4-dioctyloxyphenylhydrazine (1a) focused on deprotection of the trichloroethyl ester 3a under buffered reductive conditions, according to the general literature procedure [24]. In aqueous $\mathrm{MeOH}$ hydrazide 3a was practically insoluble, and the reaction mixture was triphasic. Under these conditions no formation of hydrazine 1a was observed. Changing $\mathrm{MeOH}$ to $\mathrm{EtOH}$ and increasing its volume by two-fold gave homogenous solutions within which the desired hydrazine 1a was formed along with significant quantities of $\mathbf{4}$ as the major products (Scheme 1). The deamination product 4 was isolated and identified by comparison with the authentic sample. The yield and proportions of the two products, $1 \mathrm{a}$ and $\mathbf{4}$, varied from run to run, according to the ${ }^{1} \mathrm{H}$ NMR spectra. Therefore, we focused on the acid-catalyzed deprotection of Boc-substituted hydrazines (Scheme 2), hydrazides 2 , expecting that the reaction could be performed under fully homogenous conditions.

Analysis of the reaction mechanism for the deprotection of 2 under acidic conditions shows that removal of the Boc group generates $t-\mathrm{Bu}^{+}$, which reacts with the solvent, or alternatively it can alkylate the benzene ring of arylhydrazine (Scheme 2). For less reactive arylhydrazines the former process is faster, $k_{1}<<k_{2}$, and deprotection with $\mathrm{HCl}$ in $\mathrm{PrOH}$ is effective [16]. For dialkoxyphenylhydrazines apparently $k_{1}>>k_{2}$ and the desired hydrazine is not obtained [16]. 


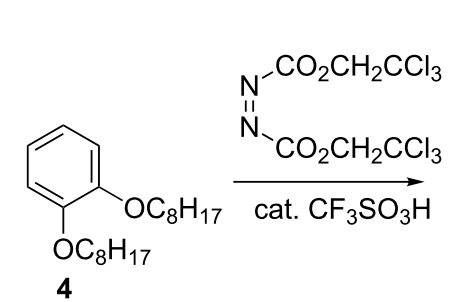

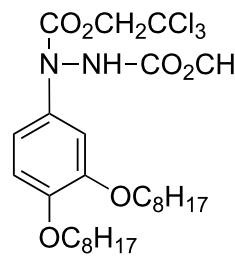

3a

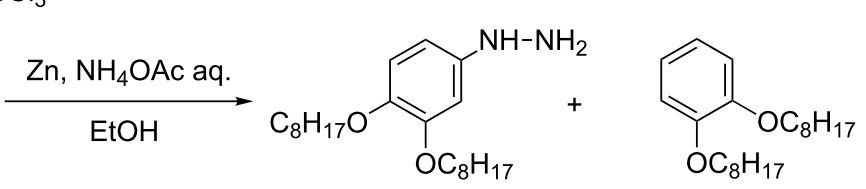

$1 \mathbf{a}$

Scheme 1: Formation and deprotection of $3 \mathbf{a}$ under reductive conditions.

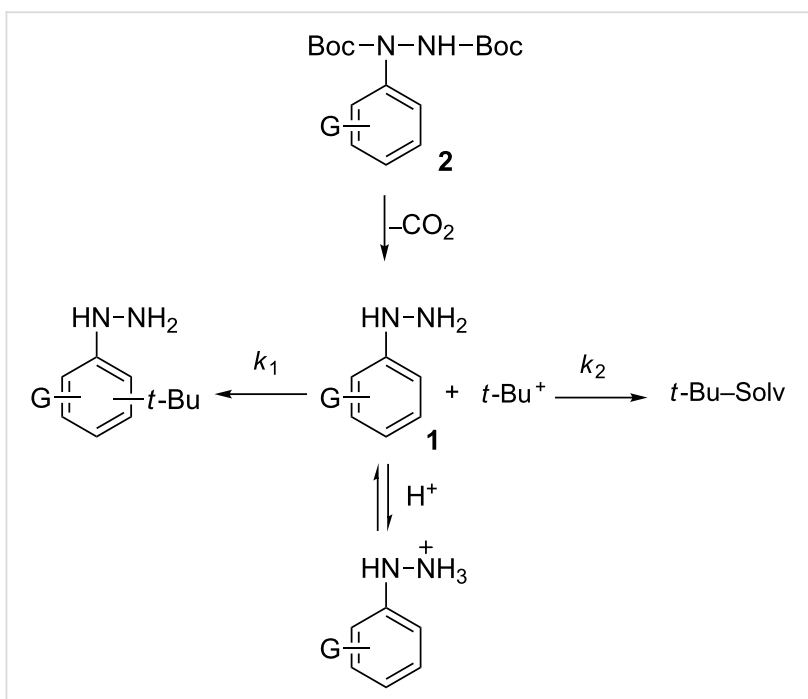

Scheme 2: General mechanism for the deprotection of arylhydrazides. $\mathrm{G}$ represents a substituent.

The nucleophilicity of the hydrazine can be suppressed by its fast and complete protonation with a strong acid (Scheme 2). In this situation, the transient $t-\mathrm{Bu}^{+}$is trapped with the solvent, forming volatile products, which simplifies isolation of the hydrazine as a crude product. We have focused on this approach to arylhydrazines employing trifluoromethanesulfonic acid ( $\mathrm{TfOH})$, which was used as an effective catalyst in the deprotection of tert-butyl aryl ethers [27].

Addition of catalytic amounts of the $\mathrm{TfOH}$ acid (10 mol \%) to solutions of hydrazide 2a (Figure 3 ) in a mixture of $\mathrm{CF}_{3} \mathrm{CH}_{2} \mathrm{OH} / \mathrm{CH}_{2} \mathrm{Cl}_{2}$ at $-40{ }^{\circ} \mathrm{C}$ gave little conversion to hydrazine 1a. With 1.5 equiv of $\mathrm{TfOH}$, hydrazide $\mathbf{2 a}$ was only<smiles>CCCCOC(=O)NN(C(=O)OCCCCCC(C)(C)C)c1ccc(OCCCC)c(OCCC)c1</smiles>

Figure 3: Structure of hydrazide $\mathbf{2 a}$. partially converted to hydrazine 1a. With 5 equiv of TfOH the reaction was complete in less than $2 \mathrm{~min}$ and the crude hydrazine 1a was isolated as the sole product. Reaction times under 2 min appear to be optimum; the purity of the hydrazine decreased with increasing reaction times.

By using this protocol, hydrazines $\mathbf{1}$ were isolated as viscous oils in purities $>90 \%$ and yields of $60-86 \%$, according to ${ }^{1} \mathrm{H}$ NMR analysis with 1,4-dimethoxybenzene as the internal standard (Scheme 3). Attempts at the preparation of crystalline hydrochlorides of $\mathbf{1}$ were unsuccessful and the viscous salts rapidly darkened and decomposed.<smiles>[X]c1cc(Br)cc([X])c1[X]</smiles>

Scheme 3: Synthesis of arylhydrazines 1. Substituents $X$ and $Y$ are defined in Figure 2.

The Boc-protected arylhydrazines, hydrazides $\mathbf{2}$, were conveniently obtained by direct addition of aryllithium to di-tert-butyl azodicarboxylate (DTBAD, Scheme 3). The latter was prepared by lithiation of aryl bromides 5 with $t$-BuLi to avoid the formation of $n$-BuBr with $n$-BuLi and N-butylation of hydrazide 2 . Hydrazide $2 \mathrm{a}$ was also obtained by the $\mathrm{Cu}^{2+}$-catalyzed addition [18] of arylboronic acid 6a [28] to DTBAD. The yields of both syntheses of $\mathbf{2 a}$ were comparable. 
The trichloroethyl hydrazide 3a was prepared by acid-catalyzed amination of 1,2-dioctyloxybenzene (4) with BTCEAD in the presence of catalytic amounts of $\mathrm{TfOH}$, according to a general literature procedure [23] (Scheme 1).

The requisite bromobenzene 5a was prepared by bromination of 1,2-dioctyloxybenzene (4) [29] with $\mathrm{CuBr}_{2}$ in $\mathrm{MeCN}$ according to a literature method [30] (Scheme 4). This method is a convenient alternative to the alkylation of the less readily accessible 4-bromocatechol (7) [28].

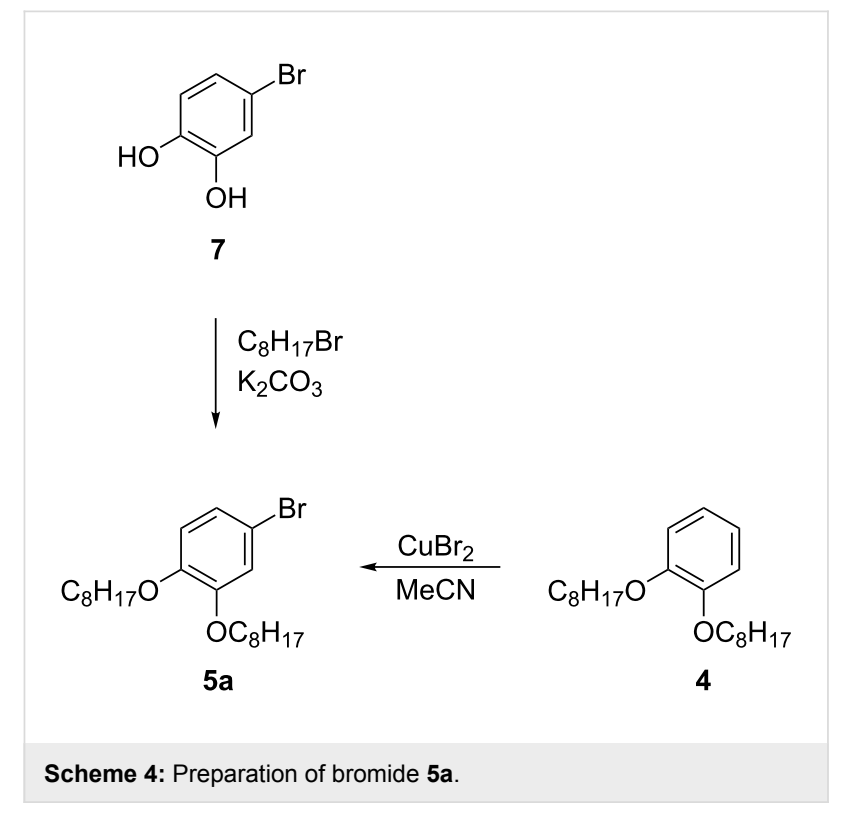

1-Bromo-3,4-didecylbenzene (5b) was obtained by bromination of 1,2-didecylbenzene (8) [31], obtained by the Kumada method [32], with $\mathrm{Br}_{2}$ in acetic anhydride (Scheme 5). Typically, the electrophilic bromination of 1,2-dialkylbenzenes results in 4,5-dibromo derivatives as the major products [33,34]. In contrast, the present method permits selective monobromination, although the bromo derivative $\mathbf{5 b}$ was isolated only in about $85 \%$ purity. The product could not be purified rigorously from several unidentified contaminants either by chromatography or by distillation due to the lack of separation or partial decomposition. Therefore, crude $\mathbf{5 b}$ was used for the preparation of hydrazide $\mathbf{2} \mathbf{b}$, which was easily purified by chromatographic methods.

The attempted monoiodination of $\mathbf{8}$ with BTMA $\cdot \mathrm{ICl}_{2}$ by using a general literature method [35] gave only traces of the product and nearly all of the starting material was recovered. Iodination under the Kern conditions [36,37] $\left(\mathrm{HIO}_{3} / \mathrm{I}_{2}\right)$ gave a mixture of mono- and diiodo derivatives, which were difficult to separate. Manipulation of the reaction time and temperature failed to give the desired monoiodo derivative as the major product.

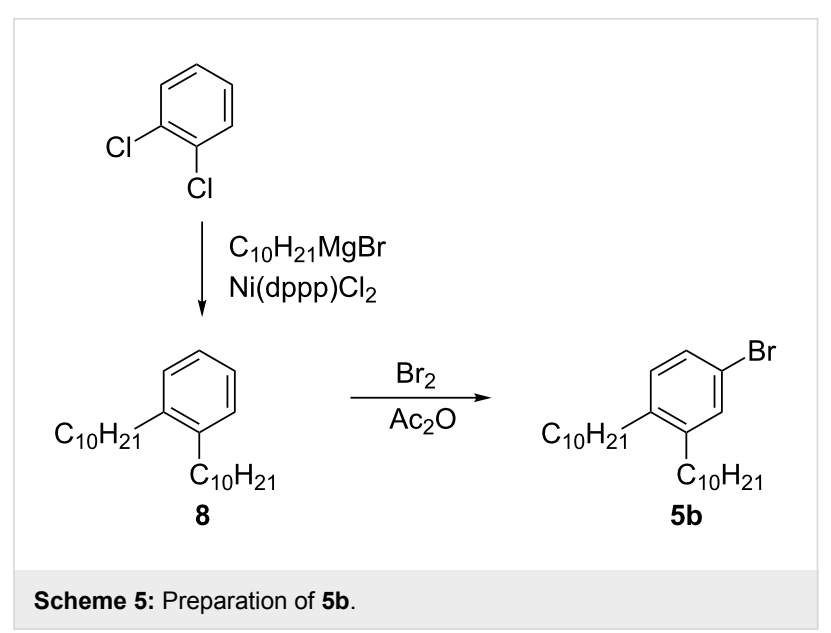

The preparation of bromobenzenes substituted with alkylsulfanyl groups, $\mathbf{5 c - 5 f}$, is described elsewhere [38]. Bromides $\mathbf{5 g}$ $[39,40]$ and $\mathbf{5 h}[41]$ were obtained according to the respective literature procedures by alkylation of 5-bromopyrogallol.

The 3,4,5-trialkylsulfanylphenylhydrazines $\mathbf{1 c}-\mathbf{1 f}$ have been used in the preparation of 6-oxoverdazyl derivatives that exhibit liquid-crystalline properties [26]. For instance, radical 9 , prepared from 1d (Figure 4), exhibits a monotropic columnar rectangular phase $\left(\mathrm{Cr} 62\left(\mathrm{Col}_{\mathrm{r}} 60\right) \mathrm{I}\right)$, a broad absorption band in the visible region, and redox potentials $\mathrm{E}^{0 /+1}{ }_{1 / 2}=+0.99 \mathrm{~V}$ and $\mathrm{E}^{0 /-1}{ }_{1 / 2}=-0.45 \mathrm{~V}$ versus SCE. Photovoltaic studies of 9 demonstrated hole mobility $\mu_{\mathrm{h}}=1.52 \times 10^{-3} \mathrm{~cm}^{2} \mathrm{~V}^{-1} \mathrm{~s}^{-1}$ in the mesophase with an activation energy $E_{\mathrm{a}}=0.06 \pm 0.01 \mathrm{eV}$.

\section{Conclusion}

We have developed a synthetic protocol for the efficient preparation of electron-rich phenylhydrazines $\mathbf{1}$ substituted with alkylsulfanyl, alkyl and alkoxy groups from Boc hydrazides $\mathbf{2}$. Experiments demonstrate that the addition of hydrazides $\mathbf{2}$ to a large excess of $\mathrm{TfOH}$ ( 5 equiv) at $-40{ }^{\circ} \mathrm{C}$ gives hydrazines $\mathbf{1}$ in yields ranging from $60-86 \%$ and with purity $>90 \%$, which is sufficient for subsequent chemical transformations. The optimum reaction time is less than $2 \mathrm{~min}$, typically $90 \mathrm{sec}$, and longer times lead to a lower purity of the product.

The presented method for the preparation of phenylhydrazines is an attractive alternative to Leblanc's method, which relies on the reductive deprotection of trichloroethyl hydrazide $\mathbf{3}$ under heterogenous conditions. Our method involves homogenous solutions, low temperatures and short reaction times, and is particularly suited to oleophilic ("greasy") arylhydrazines such as $\mathbf{1}$, which are important intermediates for the preparation of verdazyls and other heterocycles that may exhibit, e.g., liquidcrystalline properties (e.g., 9). In comparison with Leblanc's protocol, our method is also a regiocontrolled hydrazinylation 

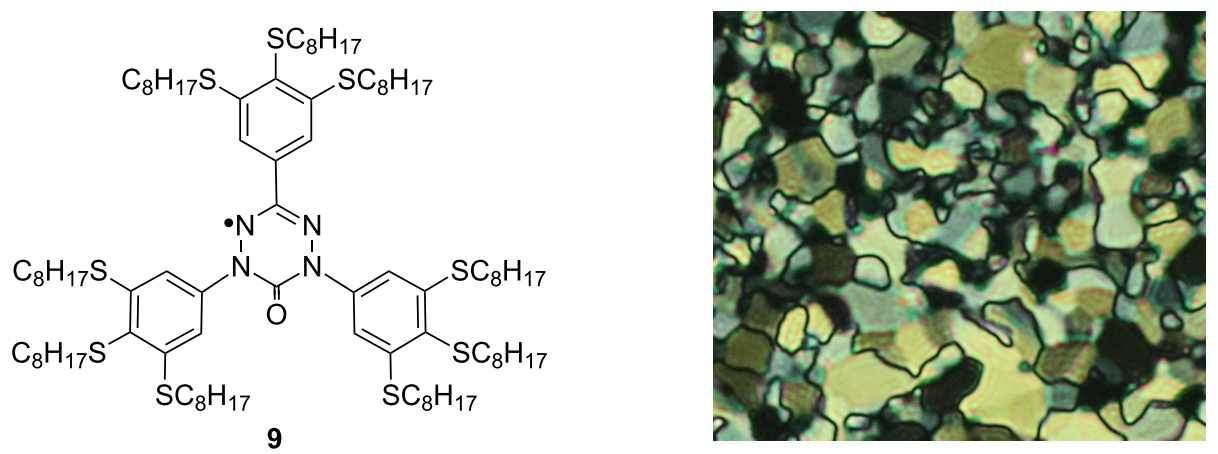

Figure 4: The structure of verdazyl radical 9 and a texture of the $\mathrm{Col}_{\mathrm{r}}$ phase.

of the aromatics with the more accessible DTBAD through the organolithium. Although we focus on long-chain-substituted phenylhydrazines, we believe that this method can be used for other electron-rich arylhydrazines.

\section{Experimental}

Reagents and solvents were obtained commercially. Reactions were carried out under Ar. ${ }^{1} \mathrm{H}$ NMR spectra were obtained at $400 \mathrm{MHz}$ in $\mathrm{CDCl}_{3}$ and referenced to the solvent, unless specified otherwise.

\section{Arylhydrazines 1}

\section{General procedure}

A solution of hydrazide $2(1 \mathrm{mmol})$ in a mixture of $\mathrm{CH}_{2} \mathrm{Cl}_{2}$ $(3 \mathrm{~mL}) / \mathrm{CF}_{3} \mathrm{CH}_{2} \mathrm{OH}(1 \mathrm{~mL})$ was rapidly added to a solution of TfOH $(0.750 \mathrm{~g}, 0.44 \mathrm{~mL}, 5 \mathrm{mmol})$ in $\mathrm{CF}_{3} \mathrm{CH}_{2} \mathrm{OH}(1 \mathrm{~mL})$ at $-40{ }^{\circ} \mathrm{C}$ under Ar. The mixture was stirred for $1.5 \mathrm{~min}$, and $\mathrm{CH}_{2} \mathrm{Cl}_{2}(5 \mathrm{~mL})$ followed by sat. $\mathrm{NaHCO}_{3}(10 \mathrm{~mL})$ were added under very vigorous stirring. The organic layer was separated and the aqueous layer extracted $\left(3 \times \mathrm{CH}_{2} \mathrm{Cl}_{2}\right)$. Then the extracts were dried $\left(\mathrm{Na}_{2} \mathrm{SO}_{4}\right)$ and the solvents were evaporated to give crude arylhydrazine 1 in purities typically $>90 \%$ as a viscous, yellow to orange oil that darkened upon standing. The quantitative analysis of the deprotection reaction was conducted with $0.2 \mathrm{mmol}$ of 2 as described above. The yield of the hydrazines was established by adding known quantities of 1,4-dimethoxybenzene ( $2.0 \mathrm{~mL}$ of $25 \mathrm{mM}$ solution in $\mathrm{CH}_{2} \mathrm{Cl}_{2}, 0.05 \mathrm{mmol}$ ) to the $\mathrm{CH}_{2} \mathrm{Cl}_{2}$ extract, evaporation of the resulting solution, and integration of the low-field ${ }^{1} \mathrm{H}$ NMR signals.

3,4-Dioctyloxyphenylhydrazine (1a): ${ }^{1} \mathrm{H}$ NMR (400 MHz, $\left.\mathrm{CDCl}_{3}\right) \delta 0.88(\mathrm{t}, J=6.8 \mathrm{~Hz}, 6 \mathrm{H}), 1.26-1.36(\mathrm{~m}, 16 \mathrm{H})$, $1.37-1.47(\mathrm{~m}, 4 \mathrm{H}), 1.70-1.85(\mathrm{~m}, 4 \mathrm{H}), 3.92(\mathrm{t}, J=6.7 \mathrm{~Hz}, 2 \mathrm{H})$, $3.96(\mathrm{t}, J=6.7 \mathrm{~Hz}, 2 \mathrm{H}), 6.34\left(\mathrm{dd}, J_{1}=8.5 \mathrm{~Hz}, J_{2}=2.6 \mathrm{~Hz}, 1 \mathrm{H}\right)$, $6.46(\mathrm{~d}, J=2.6 \mathrm{~Hz}, 1 \mathrm{H}), 6.82(\mathrm{~d}, J=8.5 \mathrm{~Hz}, 1 \mathrm{H}) ;{ }^{1} \mathrm{H} \mathrm{NMR}$ $\left(500 \mathrm{MHz}, \mathrm{DMSO}-d_{6}\right) \delta 0.86(\mathrm{t}, J=6.7 \mathrm{~Hz}, 6 \mathrm{H}), 1.20-1.36(\mathrm{~m}$,
$16 \mathrm{H}), 1.37-1.46$ (m, 4H), 1.61 (quint, $J=7.0 \mathrm{~Hz}, 2 \mathrm{H}$ ), 1.68 (quint, $J=6.9 \mathrm{~Hz}, 2 \mathrm{H}), 3.78(\mathrm{t}, J=6.4 \mathrm{~Hz}, 2 \mathrm{H}), 3.87(\mathrm{t}, J=6.3$ $\mathrm{Hz}, 2 \mathrm{H}), 6.24\left(\mathrm{dd}, J_{1}=8.5 \mathrm{~Hz}, J_{2}=2.3 \mathrm{~Hz}, 1 \mathrm{H}\right), 6.47(\mathrm{~d}, J=$ $2.3 \mathrm{~Hz}, 1 \mathrm{H}), 6.71(\mathrm{~d}, J=8.6 \mathrm{~Hz}, 1 \mathrm{H})$.

3,4-Didecylphenylhydrazine (1b): ${ }^{1} \mathrm{H}$ NMR $(500 \mathrm{MHz}$, $\left.\mathrm{CDCl}_{3}\right) \delta 0.88(\mathrm{t}, J=6.9 \mathrm{~Hz}, 6 \mathrm{H}), 1.22-1.40(\mathrm{~m}, 28 \mathrm{H})$, $1.47-1.58(\mathrm{~m}, 4 \mathrm{H}), 2.51(\mathrm{t}, J=7.0 \mathrm{~Hz}, 2 \mathrm{H}), 2.53(\mathrm{t}, J=7.1 \mathrm{~Hz}$, 2H), 2.6 (brs, 3H), 6.59-6.65 (m, 2H), 7.01 (d, $J=8.0 \mathrm{~Hz}, 1 \mathrm{H})$.

3,4,5-Trihexylsulfanylphenylhydrazine (1c): ${ }^{1} \mathrm{H}$ NMR (400 $\left.\mathrm{MHz} \mathrm{CDCl}_{3}\right) \delta 0.87(\mathrm{t}, J=6.9 \mathrm{~Hz}, 3 \mathrm{H}), 0.89(\mathrm{t}, J=6.8 \mathrm{~Hz}$, $6 \mathrm{H}), 1.20-1.35(\mathrm{~m}, 12 \mathrm{H}), 1.36-1.52(\mathrm{~m}, 6 \mathrm{H}), 1.59$ (quint, $J=$ $7.5 \mathrm{~Hz}, 2 \mathrm{H}$ ), 1.71 (quint, $J=7.4 \mathrm{~Hz}, 4 \mathrm{H}$ ), 2.77 (t, $J=7.4 \mathrm{~Hz}$, $2 \mathrm{H}), 2.83$ (t, $J=7.3 \mathrm{~Hz}, 4 \mathrm{H}), 3.2$ (brs, 3H), 6.41 (s, 2H).

3,4,5-Trioctylsulfanylphenylhydrazine (1d): ${ }^{1} \mathrm{H}$ NMR (500 $\left.\mathrm{MHz}, \mathrm{CDCl}_{3}\right) \delta 0.87(\mathrm{t}, J=6.9 \mathrm{~Hz}, 3 \mathrm{H}), 0.88(\mathrm{t}, J=6.6 \mathrm{~Hz}$, $6 \mathrm{H}), 1.20-1.34(\mathrm{~m}, 24 \mathrm{H}), 1.38-1.43(\mathrm{~m}, 2 \mathrm{H}), 1.44-1.53(\mathrm{~m}$, $4 \mathrm{H}$ ), 1.59 (quint, $J=7.5 \mathrm{~Hz}, 2 \mathrm{H}$ ), 1.72 (quint, $J=7.5 \mathrm{~Hz}, 4 \mathrm{H}$ ), 2.77 (t, $J=7.5 \mathrm{~Hz}, 2 \mathrm{H}), 2.84$ (t, $J=7.4 \mathrm{~Hz}, 4 \mathrm{H}), 6.40$ (s, 2H).

3,4,5-Tridecylsulfanylphenylhydrazine (1e): ${ }^{1} \mathrm{H}$ NMR (400 $\left.\mathrm{MHz}, \mathrm{CDCl}_{3}\right) \delta 0.87(\mathrm{t}, J=6.8 \mathrm{~Hz}, 3 \mathrm{H}), 0.88(\mathrm{t}, J=6.8 \mathrm{~Hz}$, $6 \mathrm{H}), 1.20-1.35(\mathrm{~m}, 36 \mathrm{H}), 1.36-1.52(\mathrm{~m}, 6 \mathrm{H}), 1.59$ (quint, $J=$ $7.6 \mathrm{~Hz}, 2 \mathrm{H}$ ), 1.71 (quint, $J=7.3 \mathrm{~Hz}, 4 \mathrm{H}), 2.76(\mathrm{t}, J=7.5 \mathrm{~Hz}$, 2H), 2.83 (t, $J=7.3 \mathrm{~Hz}, 4 \mathrm{H}), 6.40$ (s, 2H).

3,4,5-Tridodecylsulfanylphenylhydrazine (1f): ${ }^{1} \mathrm{H}$ NMR (500 $\left.\mathrm{MHz}, \mathrm{CDCl}_{3}\right) \delta 0.88(\mathrm{t}, J=6.8 \mathrm{~Hz}, 9 \mathrm{H}), 1.20-1.35(\mathrm{~m}, 48 \mathrm{H})$, $1.36-1.51(\mathrm{~m}, 6 \mathrm{H}), 1.59$ (quint, $J=7.5 \mathrm{~Hz}, 2 \mathrm{H}), 1.71$ (quint, $J=$ $7.4 \mathrm{~Hz}, 4 \mathrm{H}), 2.77$ (t, $J=7.4 \mathrm{~Hz}, 2 \mathrm{H}), 2.84$ (t, $J=7.1 \mathrm{~Hz}, 4 \mathrm{H})$, $6.40(\mathrm{~s}, 2 \mathrm{H})$.

3,4,5-Trioctyloxyphenylhydrazine (1g): Soft yellow solid; ${ }^{1} \mathrm{H}$ NMR $\left(400 \mathrm{MHz}, \mathrm{CDCl}_{3}\right) \delta 0.88(\mathrm{t}, J=6.7 \mathrm{~Hz}, 9 \mathrm{H})$, 
$1.22-1.38(\mathrm{~m}, 24 \mathrm{H}), 1.42-1.53(\mathrm{~m}, 6 \mathrm{H}), 1.72$ (quint, $J=7.1 \mathrm{~Hz}$, 2H), 1.79 (quint, $J=7.1 \mathrm{~Hz}, 4 \mathrm{H}), 3.86(\mathrm{t}, J=6.6 \mathrm{~Hz}, 2 \mathrm{H}), 3.95$ (t, $J=6.6 \mathrm{~Hz}, 4 \mathrm{H}), 6.06(\mathrm{~s}, 2 \mathrm{H})$.

3,4,5-Tridecyloxyphenylhydrazine (1h): ${ }^{1} \mathrm{H}$ NMR (400 MHz, $\left.\mathrm{CDCl}_{3}\right) \delta 0.88(\mathrm{t}, J=6.8 \mathrm{~Hz}, 9 \mathrm{H}), 1.21-1.38(\mathrm{~m}, 36 \mathrm{H})$, $1.39-1.64(\mathrm{~m}, 6 \mathrm{H}), 1.65-1.84(\mathrm{~m}, 6 \mathrm{H}), 3.86(\mathrm{t}, J=6.6 \mathrm{~Hz}, 2 \mathrm{H})$, $3.95(\mathrm{t}, J=6.6 \mathrm{~Hz}, 4 \mathrm{H}), 6.06(\mathrm{~s}, 2 \mathrm{H}) ;{ }^{1} \mathrm{H}$ NMR $(400 \mathrm{MHz}$, $\left.\mathrm{C}_{6} \mathrm{D}_{6}\right) \delta 0.92(\mathrm{t}, J=6.8 \mathrm{~Hz}, 9 \mathrm{H}), 1.22-1.58(\mathrm{~m}, 38 \mathrm{H}), 1.63-1.73$ (m, 4H), 1.78 (quint, $J=7.1 \mathrm{~Hz}, 4 \mathrm{H}$ ), 1.97 (quint, $J=8.3 \mathrm{~Hz}$, $2 \mathrm{H}), 3.89(\mathrm{t}, J=6.4 \mathrm{~Hz}, 4 \mathrm{H}), 4.23(\mathrm{t}, J=6.5 \mathrm{~Hz}, 2 \mathrm{H}), 6.03$ (s, $2 \mathrm{H})$.

\section{Preparation of hydrazides 2 General procedure}

To a solution of the substituted bromobenzene $5(1.0 \mathrm{mmol})$ in dry THF $(10 \mathrm{~mL}), t$-BuLi $(1.7 \mathrm{M}$ in pentane, $2.2 \mathrm{mmol})$ was added under $\mathrm{Ar}$ at $-78^{\circ} \mathrm{C}$. After $1.5 \mathrm{~h}$ a THF $(1 \mathrm{~mL})$ solution of di-tert-butyl azodicarboxylate (DTBAD, $345 \mathrm{mg}, 1.5 \mathrm{mmol}$ ) was added dropwise. The mixture was stirred at $-78{ }^{\circ} \mathrm{C}$ for $0.5 \mathrm{~h}$, then $1 \mathrm{~h}$ at $\mathrm{rt}$, and quenched with $5 \% \mathrm{HCl}$. The organic products were extracted $\left(\mathrm{Et}_{2} \mathrm{O}\right)$, the extracts dried $\left(\mathrm{Na}_{2} \mathrm{SO}_{4}\right)$, the solvents evaporated, and the residue was passed through a short silica-gel column (hexane/ $\mathrm{CH}_{2} \mathrm{Cl}_{2}$ then $\mathrm{CH}_{2} \mathrm{Cl}_{2}$ ) to give hydrazides 2 as white solids.

1,2-Bis(tert-butoxycarbonyl)-1-(3,4-dioctyloxyphenyl)hydrazine (2a): Yield 71\%; mp 55-57 ${ }^{\circ} \mathrm{C} ;{ }^{1} \mathrm{H}$ NMR $(500 \mathrm{MHz}$, $\left.\mathrm{CDCl}_{3}\right) \delta 0.88(\mathrm{t}, J=6.9 \mathrm{~Hz}, 6 \mathrm{H}), 1.22-1.38(\mathrm{~m}, 16 \mathrm{H})$, $1.39-1.51(\mathrm{~m}, 4 \mathrm{H}), 1.49(\mathrm{~s}, 18 \mathrm{H}), 1.73-1.84(\mathrm{~m}, 4 \mathrm{H}), 3.96(\mathrm{t}, J$ $=6.6 \mathrm{~Hz}, 2 \mathrm{H}), 3.97(\mathrm{t}, J=6.6 \mathrm{~Hz}, 2 \mathrm{H}), 6.71(\mathrm{brs}, 1 \mathrm{H}), 6.80(\mathrm{~d}, J$ $=8.6 \mathrm{~Hz}, 1 \mathrm{H}), 6.86-6.92(\mathrm{~m}, 1 \mathrm{H}), 6.93-7.02(\mathrm{~m}, 1 \mathrm{H})$; Anal. calcd for $\mathrm{C}_{32} \mathrm{H}_{56} \mathrm{~N}_{2} \mathrm{O}_{6}$ : C, 68.05; H, 9.99; N, 4.96; found: $\mathrm{C}$, 68.35; H, 9.82; N, 5.02.

Method B: To a solution of 3,4-dioctyloxyphenylboronic acid (6a, $50 \mathrm{mg}, 0.13 \mathrm{mmol})$ in THF $(2 \mathrm{~mL})$, di-tert-butyl azodicarboxylate (DTBAD, $30 \mathrm{mg}, 0.13 \mathrm{mmol}$ ) was added followed by $\mathrm{Cu}(\mathrm{OAc})_{2}$ (cat) under an $\mathrm{Ar}$ atmosphere. The mixture was stirred at rt overnight, the solvent was evaporated and the residue was purified on a short silica-gel column $\left(\mathrm{CH}_{2} \mathrm{Cl}_{2}\right)$ to give $50 \mathrm{mg}$ (68\% of yield) of hydrazide $\mathbf{2 a}$.

1,2-Bis(tert-butoxycarbonyl)-1-(3,4-didecylphenyl)hydrazine (2b): Yield 63\%; mp 37-38 ${ }^{\circ} \mathrm{C}$; ${ }^{1} \mathrm{H} \mathrm{NMR}\left(500 \mathrm{MHz}, \mathrm{CDCl}_{3}\right) \delta$ 0.88 (t, $J=6.8 \mathrm{~Hz}, 6 \mathrm{H}), 1.23-1.40(\mathrm{~m}, 28 \mathrm{H}), 1.49$ (s, 18H), $1.48-1.59(\mathrm{~m}, 4 \mathrm{H}), 2.52-2.59(\mathrm{~m}, 4 \mathrm{H}), 6.70$ (brs, $1 \mathrm{H}), 7.06$ (d, $J$ $=8.2 \mathrm{~Hz}, 1 \mathrm{H}), 7.08-7.21$ (br $\mathrm{m}, 2 \mathrm{H})$; Anal. calcd for $\mathrm{C}_{36} \mathrm{H}_{64} \mathrm{~N}_{2} \mathrm{O}_{4}$ : C, 73.42; H, 10.95; N, 4.76; found: C, 73.06; $\mathrm{H}$, $10.88 ; \mathrm{N}, 4.74$.
1,2-Bis(tert-butoxycarbonyl)-1-(3,4,5-trihexylsulfanylphenyl)hydrazine (2c): Yield 43\%; mp $74-75{ }^{\circ} \mathrm{C} ;{ }^{1} \mathrm{H}$ NMR $\left(400 \mathrm{MHz} \mathrm{CDCl}_{3}\right) \delta 0.87(\mathrm{t}, J=6.9 \mathrm{~Hz}, 3 \mathrm{H}), 0.88(\mathrm{t}, J=6.9$ $\mathrm{Hz}, 6 \mathrm{H}), 1.23-1.37(\mathrm{~m}, 12 \mathrm{H}), 1.38-1.52(\mathrm{~m}, 6 \mathrm{H}), 1.51(\mathrm{~s}, 18 \mathrm{H})$, 1.60 (quint, $J=7.4 \mathrm{~Hz}, 2 \mathrm{H}$ ), 1.72 (quint, $J=7.2 \mathrm{~Hz}, 4 \mathrm{H}$ ), 2.81 (t, $J=7.5 \mathrm{~Hz}, 2 \mathrm{H}), 2.84$ (t, $J=7.4 \mathrm{~Hz}, 4 \mathrm{H}), 6.69$ (brs, 1H), 6.99 (brs, $2 \mathrm{H}$ ); Anal. calcd for $\mathrm{C}_{34} \mathrm{H}_{60} \mathrm{~N}_{2} \mathrm{O}_{4} \mathrm{~S}_{3}$ : C, 62.15; H, 9.20; N, 4.26; found: C, 62.35; H, 9.34; N, 4.22.

1,2-Bis(tert-butoxycarbonyl)-1-(3,4,5-trioctylsulfanylphenyl)hydrazine (2d): Yield $55 \%$ yield; mp $51-52{ }^{\circ} \mathrm{C}$; ${ }^{1} \mathrm{H}$ NMR (400 MHz, $\left.\mathrm{CDCl}_{3}\right) \delta 0.84-0.91(\mathrm{~m}, 9 \mathrm{H}), 1.21-1.35$ $(\mathrm{m}, 24 \mathrm{H}), 1.36-1.50(\mathrm{~m}, 6 \mathrm{H}), 1.51(\mathrm{~s}, 18 \mathrm{H}), 1.60$ (quint, $J=7.4$ $\mathrm{Hz}, 2 \mathrm{H}$ ), 1.72 (quint, $J=7.5 \mathrm{~Hz}, 4 \mathrm{H}$ ), 2.81 (t, $J=7.5 \mathrm{~Hz}, 4 \mathrm{H}$ ), 2.83 (t, $J=7.4 \mathrm{~Hz}, 2 \mathrm{H}$ ), 6.69 (brs, 1H), 6.98 (brs, 2H); the analytically pure sample was obtained by recrystallization (MeCN); Anal. calcd for $\mathrm{C}_{40} \mathrm{H}_{72} \mathrm{~N}_{2} \mathrm{O}_{4} \mathrm{~S}_{3}$ : C, 64.82; H, 9.79; N, 3.78; found: C, 64.92; H, 9.56; N, 3.91 .

1,2-Bis(tert-butoxycarbonyl)-1-(3,4,5-tridecylsulfanylphenyl)hydrazine (2e): Yield 56\%; mp 50-51 ${ }^{\circ} \mathrm{C} ;{ }^{1} \mathrm{H}$ NMR $\left(500 \mathrm{MHz}, \mathrm{CDCl}_{3}\right) \delta 0.87(\mathrm{t}, J=6.8 \mathrm{~Hz}, 3 \mathrm{H}), 0.88(\mathrm{t}, J=7.0$ $\mathrm{Hz}, 6 \mathrm{H}), 1.21-1.36(\mathrm{~m}, 38 \mathrm{H}), 1.37-1.51(\mathrm{~m}, 6 \mathrm{H}), 1.51(\mathrm{~s}, 18 \mathrm{H})$, 1.60 (quint, $J=7.4 \mathrm{~Hz}, 2 \mathrm{H}$ ), 1.71 (quint, $J=7.4 \mathrm{~Hz}, 4 \mathrm{H}$ ), 2.81 (t, $J=7.6 \mathrm{~Hz}, 2 \mathrm{H}), 2.83$ (t, $J=7.4 \mathrm{~Hz}, 4 \mathrm{H}), 6.70$ (brs, 1H), 6.98 (brs, $2 \mathrm{H}$ ); Anal. calcd for $\mathrm{C}_{46} \mathrm{H}_{86} \mathrm{~N}_{2} \mathrm{O}_{4} \mathrm{~S}_{3}$ : C, 66.78; H, 10.48; $\mathrm{N}, 3.39$; found: C, 66.75; H, 10.07; N, 3.43.

1,2-Bis(tert-butoxycarbonyl)-1-(3,4,5-tridodecylsulfanylphenyl)hydrazine (2f): Yield 50\%; mp 49-51 ${ }^{\circ} \mathrm{C} ;{ }^{1} \mathrm{H}$ NMR $\left(500 \mathrm{MHz}, \mathrm{CDCl}_{3}\right) \delta 0.88(\mathrm{t}, J=6.7 \mathrm{~Hz}, 9 \mathrm{H}), 1.22-1.36(\mathrm{~m}$, $50 \mathrm{H}), 1.35-1.51(\mathrm{~m}, 6 \mathrm{H}), 1.51(\mathrm{~s}, 18 \mathrm{H}), 1.60$ (quint, $J=7.4 \mathrm{~Hz}$, 2H), 1.71 (quint, $J=7.2 \mathrm{~Hz}, 4 \mathrm{H}$ ), 2.81 (t, $J=7.4 \mathrm{~Hz}, 2 \mathrm{H}$ ), 2.83 (t, $J=7.2 \mathrm{~Hz}, 4 \mathrm{H}), 6.69$ (brs, 1H), 6.98 (brs, 2H); Anal. calcd for $\mathrm{C}_{52} \mathrm{H}_{98} \mathrm{~N}_{2} \mathrm{O}_{4} \mathrm{~S}_{3}: \mathrm{C}, 68.52 ; \mathrm{H}, 10.84 ; \mathrm{N}, 3.07$; found: $\mathrm{C}$, $68.82 ; \mathrm{H}, 10.83 ; \mathrm{N}, 3.06$.

1,2-Bis(tert-butoxycarbonyl)-1-(3,4,5-trioctyloxyphenyl)hydrazine (2g): Yield 45\%; white crystals $(\mathrm{MeCN} /$ EtOAc); mp 73-74 ${ }^{\circ} \mathrm{C} ;{ }^{1} \mathrm{H}$ NMR $\left(400 \mathrm{MHz}, \mathrm{CDCl}_{3}\right) \delta 0.88(\mathrm{t}, J$ $=6.8 \mathrm{~Hz}, 9 \mathrm{H}), 1.23-1.38(\mathrm{~m}, 24 \mathrm{H}), 1.38-1.52(\mathrm{~m}, 6 \mathrm{H}), 1.50(\mathrm{~s}$, $18 \mathrm{H}$ ), 1.73 (quint, $J=7.4 \mathrm{~Hz}, 2 \mathrm{H}$ ), 1.77 (quint, $J=6.9 \mathrm{~Hz}, 4 \mathrm{H}$ ), $3.92(\mathrm{t}, J=6.8 \mathrm{~Hz}, 2 \mathrm{H}), 3.93(\mathrm{t}, J=6.6 \mathrm{~Hz}, 4 \mathrm{H}), 6.64$ (brs, $2 \mathrm{H})$, 6.68 (brs, $1 \mathrm{H}$ ); Anal. calcd for $\mathrm{C}_{40} \mathrm{H}_{72} \mathrm{~N}_{2} \mathrm{O}_{7}$ : C, 69.32; $\mathrm{H}$, 10.47; N, 4.04; found: C, 69.61; H, 10.43; N, 3.91 .

1,2-Bis (tert-butoxycarbonyl)-1-(3,4,5-tridecyloxyphenyl)hydrazine (2h): Yield $64 \%$; mp $55-57{ }^{\circ} \mathrm{C} ;{ }^{1} \mathrm{H}$ NMR $\left(400 \mathrm{MHz}, \mathrm{CDCl}_{3}\right) \delta 0.88(\mathrm{t}, J=6.8 \mathrm{~Hz}, 9 \mathrm{H}), 1.21-1.38(\mathrm{~m}$, $38 \mathrm{H}), 1.41-1.54(\mathrm{~m}, 6 \mathrm{H}), 1.50(\mathrm{~s}, 18 \mathrm{H}), 1.67-1.82(\mathrm{~m}, 6 \mathrm{H})$, $3.91(\mathrm{t}, J=6.8 \mathrm{~Hz}, 2 \mathrm{H}), 3.93(\mathrm{t}, J=6.6 \mathrm{~Hz}, 4 \mathrm{H}), 6.64$ (brs, 2H), 
6.68 (brs, $1 \mathrm{H})$; Anal. calcd for $\mathrm{C}_{46} \mathrm{H}_{86} \mathrm{~N}_{2} \mathrm{O}_{7}$ : C, 70.91; $\mathrm{H}$, 11.12; N, 3.60; found: C, 71.31; H, 11.08; N, 3.65.

1,2-Bis(2,2,2-trichloroethoxycarbonyl)-1-(3,4-dioctyloxyphenyl)hydrazine (3a): To the solution of 1,2-dioctyloxybenzene $(4,1.10 \mathrm{~g}, 3.31 \mathrm{mmol})$ in dry $\mathrm{CH}_{2} \mathrm{Cl}_{2}(20 \mathrm{~mL})$, one drop of $\mathrm{CF}_{3} \mathrm{SO}_{3} \mathrm{H}$ was added under $\mathrm{Ar}$ at $-78{ }^{\circ} \mathrm{C}$ followed by a solution of bis(2,2,2-trichloroethyl) azodicarboxylate (BTCEAD, $1.50 \mathrm{~g}, 3.97 \mathrm{mmol})$ in $\mathrm{CH}_{2} \mathrm{Cl}_{2}(3 \mathrm{~mL})$. The reaction mixture was stirred for $20 \mathrm{~min}$, warmed up to rt, stirred for $10 \mathrm{~min}$, and quenched with $25 \% \mathrm{NH}_{4} \mathrm{OAc}$. The organic products were extracted $\left(\mathrm{CH}_{2} \mathrm{Cl}_{2}\right)$, the extracts dried $\left(\mathrm{Na}_{2} \mathrm{SO}_{4}\right)$, and the solvent evaporated. The viscous residue was passed through a silica-gel plug (hexane $/ \mathrm{CH}_{2} \mathrm{Cl}_{2}$ then $\mathrm{CH}_{2} \mathrm{Cl}_{2}$ ) to give $1.03 \mathrm{~g}$ (36\% yield) of the hydrazide $3 \mathbf{a}$ as a viscous oil: ${ }^{1} \mathrm{H}$ NMR (400 $\left.\mathrm{MHz}_{\mathrm{CDCl}}\right) \delta 0.88(\mathrm{t}, J=6.7 \mathrm{~Hz}, 3 \mathrm{H}), 0.89(\mathrm{t}, J=7.2 \mathrm{~Hz}$, $3 \mathrm{H}), 1.24-1.39(\mathrm{~m}, 16 \mathrm{H}), 1.41-1.50(\mathrm{~m}, 4 \mathrm{H}), 1.80$ (quint, $J=$ $7.0 \mathrm{~Hz}, 4 \mathrm{H}), 3.96(\mathrm{t}, J=6.6 \mathrm{~Hz}, 2 \mathrm{H}), 3.99(\mathrm{t}, J=6.6 \mathrm{~Hz}, 2 \mathrm{H})$, $4.82(\mathrm{~s}, 4 \mathrm{H}), 6.83(\mathrm{~d}, J=8.6 \mathrm{~Hz}, 1 \mathrm{H}), 6.97(\mathrm{~d}, J=8.2 \mathrm{~Hz}, 1 \mathrm{H})$, 7.02 (brs, $1 \mathrm{H}$ ), 7.39 (brs, $1 \mathrm{H}$ ); Anal. calcd for $\mathrm{C}_{28} \mathrm{H}_{42} \mathrm{Cl}_{6} \mathrm{~N}_{2} \mathrm{O}_{6}$ : C, 47.01; H, 5.92; N, 3.92; found: C, 46.27; H, 5.72; N, 3.92 .

1-Bromo-3,4-didecylbenzene (5b): To a solution of 1,2-didecylbenzene $(8,1.00 \mathrm{~g}, 2.8 \mathrm{mmol})$ in a mixture of $\mathrm{Ac}_{2} \mathrm{O}(3 \mathrm{~mL})$ and $\mathrm{CH}_{2} \mathrm{Cl}_{2}(3 \mathrm{~mL}), \mathrm{Br}_{2}(0.30 \mathrm{~mL}, 5.6 \mathrm{mmol})$ and catalytic amounts of $\mathrm{I}_{2}$ were added. The reaction mixture was stirred overnight at rt, water was added, the organic products were extracted (hexane), the extracts dried $\left(\mathrm{Na}_{2} \mathrm{SO}_{4}\right)$, and the solvents evaporated. The residue was passed through a silicagel plug (hexane) to give $1.20 \mathrm{~g}$ ( $\sim 85 \%$ yield, based on NMR, contained $\sim 15 \%$ of at least two impurities) of 4-bromo-1,2-didecylbenzene (5b) as a colorless oil: ${ }^{1} \mathrm{H} \mathrm{NMR}\left(500 \mathrm{MHz}, \mathrm{CDCl}_{3}\right)$ major signals $\delta 0.88(\mathrm{t}, J=6.8 \mathrm{~Hz}, 6 \mathrm{H}), 1.20-1.40(\mathrm{~m}, 28 \mathrm{H})$, $1.49-1.58(\mathrm{~m}, 4 \mathrm{H}), 2.53(\mathrm{t}, J=7.4 \mathrm{~Hz}, 2 \mathrm{H}), 2.55(\mathrm{t}, J=7.5 \mathrm{~Hz}$, $2 \mathrm{H}), 6.98(\mathrm{~d}, J=8.2 \mathrm{~Hz}, 1 \mathrm{H}), 7.22\left(\mathrm{dd}, J_{1}=8.2 \mathrm{~Hz}, J_{2}=1.8\right.$ $\mathrm{Hz}, 1 \mathrm{H}), 7.25-7.27(\mathrm{~m}, 1 \mathrm{H}) ;{ }^{1} \mathrm{H}$ NMR $\left(400 \mathrm{MHz}, \mathrm{CD}_{2} \mathrm{Cl}_{2}\right)$ major signals $\delta 0.86(\mathrm{t}, J=6.8 \mathrm{~Hz}, 6 \mathrm{H}), 1.20-1.40(\mathrm{~m}, 28 \mathrm{H})$, $1.49-1.58(\mathrm{~m}, 4 \mathrm{H}), 2.52(\mathrm{t}, J=7.4 \mathrm{~Hz}, 2 \mathrm{H}), 2.55(\mathrm{t}, J=7.5 \mathrm{~Hz}$, $2 \mathrm{H}), 6.99(\mathrm{~d}, J=8.2 \mathrm{~Hz}, 1 \mathrm{H}), 7.19\left(\mathrm{dd}, J_{1}=8.1 \mathrm{~Hz}, J_{2}=2.2\right.$ $\mathrm{Hz}, 1 \mathrm{H}), 7.25(\mathrm{~d}, J=2.1 \mathrm{~Hz}, 1 \mathrm{H})$; HRMS-EI $(\mathrm{m} / \mathrm{z})$ : $[\mathrm{M}]^{+}$calcd for $\mathrm{C}_{26} \mathrm{H}_{45} \mathrm{Br}$, 436.2705; found, 436.2726; since $\mathbf{5 b}$ undergoes partial decomposition during attempted short-path distillation $\left(>260{ }^{\circ} \mathrm{C} / 0.2 \mathrm{mmHg}\right)$, it was used without further purification for the preparation of $\mathbf{2} \mathbf{b}$.

1,2-Didecylbenzene (8): Following a general procedure [31], a solution of 1,2-dichlorobenzene $(10.0 \mathrm{~g}, 68.0 \mathrm{mmol})$, $\mathrm{Ni}(\mathrm{dppp}) \mathrm{Cl}_{2}$ (370 mg, $\left.0.68 \mathrm{mmol}\right)$, and $n$-decylmagnesium bromide $(272 \mathrm{mmol})$ in a dry THF $(100 \mathrm{~mL})$ was heated under reflux overnight. The crude product was passed through a silicagel plug (hexane) and short-path distilled $\left(220-230{ }^{\circ} \mathrm{C} / 0.3\right.$
$\mathrm{mmHg}$ ) to collect $11.4 \mathrm{~g}$ (48\% yield) of 1,2-didecylbenzene (8) as a colorless oil: ${ }^{1} \mathrm{H}$ NMR (400 MHz, $\left.\mathrm{CDCl}_{3}\right) \delta 0.88(\mathrm{t}, J=6.8$ $\mathrm{Hz}, 6 \mathrm{H}), 1.20-1.43$ (m, 28H), 1.57 (quint, $J=7.7 \mathrm{~Hz}, 4 \mathrm{H}), 2.59$ (t, $J=8.0 \mathrm{~Hz}, 4 \mathrm{H}), 7.06-7.16(\mathrm{~m}, 4 \mathrm{H})$; HRMS-EI $(\mathrm{m} / \mathrm{z}):[\mathrm{M}]^{+}$ calcd for $\mathrm{C}_{26} \mathrm{H}_{46}, 358.3600$; found, 358.3583 .

\section{Acknowledgements}

This project was supported by a Vanderbilt University Discovery Grant.

\section{References}

1. Robinson, B. The Fisher Indole Synthesis; Wiley \& Sons: New York, 1982.

2. Elguero, J. Pyrazoles. In Comprehensive Heterocyclic Chemistry II; Katritzky, A. R.; Rees, C. W.; Scriven, E. F. V., Eds.; Pergamon, 1996; Vol. 3, pp 1-75. doi:10.1016/B978-008096518-5.00059-9

3. Garratt, P. J. 1,2,4-Triazoles. In Comprehensive Heterocyclic Chemistry II; Katritzky, A. R.; Rees, C. W.; Scriven, E. F. V., Eds.; Pergamon, 1996; Vol. 4, pp 127-163. doi:10.1016/B978-008096518-5.00080-0

4. Duffy, K. J.; Darcy, M. G.; Delorme, E.; Dillon, S. B.; Eppley, D. F.; Erickson-Miller, C.; Giampa, L.; Hopson, C. B.; Huang, Y.; Keenan, R. M.; Lamb, P.; Leong, L.; Liu, N.; Miller, S. G.; Price, A. T.; Rosen, J.; Shah, R.; Shaw, T. N.; Smith, H.; Stark, K. C.; Tian, S.-S.; Tyree, C.; Wiggall, K. J.; Zhang, L.; Luengo, J. I. J. Med. Chem. 2001, 44, 3730. doi:10.1021/jm010283I

5. He, L.; Chang, H.-X.; Chou, T.-C.; Savaraj, N.; Cheng, C. C. Eur. J. Med. Chem. 2003, 38, 101. doi:10.1016/S0223-5234(02)01420-4

6. Sugimoto, A.; Tanaka, H.; Eguchi, Y.; Ito, S.; Takashima, Y.; Ishikawa, M. J. Med. Chem. 1984, 27, 1300. doi:10.1021/jm00376a013

7. Wiley, P. F. Verdazyls. Chemistry of 1,2,3-Triazines and 1,2,4-Triazines, Tetrazines and Pentazines; The Chemistry of Heterocyclic Compounds; Willey \& Sons: New York, 1978; pp 1225-1246.

8. Koivisto, B. D.; Hicks, R. G. Coord. Chem. Rev. 2005, 249, 2612. doi:10.1016/j.ccr.2005.03.012

9. Hicks, R. G. Verdazyls and Related Radicals Containing the Hydrazyl $\left[\mathrm{R}_{2} \mathrm{~N}-\mathrm{NR}\right]$ Group. In Stable Radicals: Fundamentals and Applied Aspects of Odd-Electron Compounds; Hicks, R. G., Ed.; Wiley \& Sons, 2010; pp 245-280. And references therein.

10. Neugebauer, F. A.; Umminger, I. Chem. Ber. 1980, 113, 1205. doi:10.1002/cber.19801130402

11. Constantinides, C. P.; Koutentis, P. A.; Krassos, H.; Rawson, J. M.; Tasiopoulos, A. J. J. Org. Chem. 2011, 76, 2798. doi:10.1021/jo200210s

12. Koutentis, P. A.; Lo Re, D. Synthesis 2010, 2075. doi:10.1055/s-0029-1218782

13. Hunsberger, I. M.; Shaw, E. R.; Fugger, J.; Ketcham, R.; Lednicer, D. J. Org. Chem. 1956, 21, 394. doi:10.1021/j001110a004

14. Bandgar, B. P.; Thite, C. S. Synth. Commun. 1997, 27, 635. doi:10.1080/00397919708003336

15. Browne, D. L.; Baxendale, I. R.; Ley, S. V. Tetrahedron 2011, 67, 10296. doi:10.1016/j.tet.2011.09.146

16. Demers, J. P.; Klaubert, D. H. Tetrahedron Lett. 1987, 28, 4933. doi:10.1016/S0040-4039(00)96662-0 
17. Velarde-Ortiz, R.; Guijarro, A.; Rieke, R. D. Tetrahedron Lett. 1998, 39, 9157. doi:10.1016/S0040-4039(98)02108-X

18. Kabalka, G. W.; Guchhait, S. K. Org. Lett. 2003, 5, 4129 doi:10.1021/ol035544v

19. Uemura, T.; Chatani, N. J. Org. Chem. 2005, 70, 8631. doi:10.1021/jo051387x

20. Muñiz, K.; Iglesias, A. Angew. Chem., Int. Ed. 2007, 46, 6350. doi:10.1002/anie.200700288

21. Zaltsgendler, I.; Leblanc, Y.; Bernstein, M. A. Tetrahedron Lett. 1993, 34, 2441. doi:10.1016/S0040-4039(00)60436-7

22. Mitchell, H.; Leblanc, Y. J. Org. Chem. 1994, 59, 682. doi:10.1021/jo00082a035

23. Leblanc, Y.; Boudreault, N. J. Org. Chem. 1995, 60, 4268. doi:10.1021/jo00118a052

24. Dufresne, C.; Leblanc, Y.; Berthelette, C.; McCooeye, C. Synth. Commun. 1997, 27, 3613. doi:10.1080/00397919708007084

25. Hudlicky, T.; Seoane, G.; Pettus, T. J. Org. Chem. 1989, 54, 4239. doi:10.1021/jo00278a052

26. Jankowiak, A.; Pociecha, D.; Szczytko, J.; Monobe, H.; Kaszyński, P. J. Am. Chem. Soc. 2012, 134, 2465. doi:10.1021/ja209467h

27. Holcombe, J. L.; Livinghouse, T. J. Org. Chem. 1986, 51, 111. doi:10.1021/jo00351a028

28. Sienkowska, M. J.; Farrar, J. M.; Zhang, F.; Kusuma, S.; Heiney, P. A.; Kaszynski, P. J. Mater. Chem. 2007, 17, 1399. doi:10.1039/b615545f

29. Collins, R. F.; Davis, M. J. Chem. Soc. C 1966, 366. doi:10.1039/J39660000366

30. Bhatt, S.; Nayak, S. K. Synth. Commun. 2007, 37, 1381. doi:10.1080/00908320701230026

31. Mohr, B.; Enkelmann, V.; Wegner, G. J. Org. Chem. 1994, 59, 635. doi:10.1021/jo00082a022

32. Tamao, K.; Sumitani, K.; Kiso, Y.; Zembayashi, M.; Fujioka, A.; Kodama, S.-i.; Nakajima, I.; Minato, A.; Kumada, M. Bull. Chem. Soc. Jpn. 1976, 49, 1958. doi:10.1246/bcsj.49.1958

33. Sonoda, M.; Sakai, Y.; Yoshimura, T.; Tobe, Y.; Kamada, K. Chem. Lett. 2004, 33, 972. doi:10.1246/cl.2004.972

34. Hanack, M.; Haisch, P.; Lehmann, H.; Subramanian, L. R. Synthesis 1993, 387. doi:10.1055/s-1993-25869

35. Kajigaeshi, S.; Kakinami, T.; Moriwaki, M.; Tanaka, T.; Fujisaki, S.; Okamoto, T. Bull. Chem. Soc. Jpn. 1989, 62, 439. doi:10.1246/bcsj.62.439

36. Wirth, H. O.; Königstein, O.; Kern, W. Justus Liebigs Ann. Chem. 1960, 634, 84. doi:10.1002/jlac.19606340109

37. Jasiński, M.; Jankowiak, A.; Januszko, A.; Bremer, M.; Pauluth, D.; Kaszyński, P. Liq. Cryst. 2008, 35, 343. doi:10.1080/02678290701817318

38. Jankowiak, A.; Dębska, Z.; Romański, J.; Kaszyński, P. J. Sulfur Chem. 2012, 33. doi:10.1080/17415993.2011.644554

39. Maeda, H.; Haketa, Y.; Nakanishi, T. J. Am. Chem. Soc. 2007, 129, 13661. doi:10.1021/ja074435z

40. Yasuda, T.; Shimizu, T.; Liu, F.; Ungar, G.; Kato, T. J. Am. Chem. Soc. 2011, 133, 13437. doi:10.1021/ja2035255

41. Ma, C.-Q.; Pisula, W.; Weber, C.; Feng, X.-L.; Müllen, K.; Bäuerle, P. Chem.-Eur. J. 2011, 17, 1507. doi:10.1002/chem.201002198

\section{License and Terms}

This is an Open Access article under the terms of the Creative Commons Attribution License

(http://creativecommons.org/licenses/by/2.0), which permits unrestricted use, distribution, and reproduction in any medium, provided the original work is properly cited.

The license is subject to the Beilstein Journal of Organic Chemistry terms and conditions:

(http://www.beilstein-journals.org/bjoc)

The definitive version of this article is the electronic one which can be found at: doi:10.3762/bjoc. 8.29 